\title{
Mobility Analysis of FeTe Thin Films
}

\author{
Ichiro TSUKADA ${ }^{1,3 *}$, Masafumi HANAWA ${ }^{1,3}$, Seiki KOMIYA ${ }^{1,3}$, Ataru ICHINOSE ${ }^{1,3}$, \\ Takanori AKIIKE ${ }^{2,3}$, Yoshinori $\mathrm{IMAI}^{2,3}$, and Atsutaka MAEDA ${ }^{2,3}$ \\ ${ }^{1}$ Central Research Institute of Electric Power Industry, 2-6-1 Nagasaka, Yokosuka, Kanagawa \\ 240-0196, Japan \\ ${ }^{2}$ Department of Basic Science, The University of Tokyo, 3-8-1 Komaba, Meguro, Tokyo 153-8902, \\ Japan \\ ${ }^{3}$ JST, TRIP, Sanbancho, Chiyoda, Tokyo 102-0075, Japan
}

\begin{abstract}
The Hall effect is investigated in detail for nonsuperconducting and superconducting FeTe thin films. The Hall coefficient commonly exhibits a sign reversal from positive in a hightemperature paramagnetic state to negative in a low-temperature antiferromagnetic state. Phenomenological analysis by a simple two-band Drude model indicates that hole mobility is significantly suppressed in the antiferromagnetic state. When suppression of the hole mobility is insufficient, superconductivity shows up in FeTe. This result strongly suggests that the itinerancy in both hole and electron channels is the essential factor for the occurrence of superconductivity in iron chalcogenide superconductors.
\end{abstract}

KEYWORDS: iron chalcogenide, FeTe, Hall effect, thin films, two-band model

Iron-pnictide $^{1)}$ and iron-chalcogenide ${ }^{2)}$ superconductors are interesting materials when compared with cuprate superconductors. In both compounds, superconductivity is induced by chemical substitution to a parent antiferromagnet suggesting a possible common mechanism resulting in a similar phase diagram. However, the nature of the antiferromagnetic (AFM) state is qualitatively different. Cuprates are characterized as single-band metals and become an insulator, while iron-based superconductors are characterized as multi-band metals, ${ }^{3-5)}$ and exhibit metallic behavior even in the AFM state. ${ }^{6)}$ Actually undoped parent compounds of iron-based superconductors are compensated metals, where both electrons and holes contribute to the electrical transport. Therefore, this difference should be carefully considered when discussing the phase diagram, and it is important to understand how electrons and holes contribute to the electrical transport and superconductivity.

The multi-band nature of iron-based superconductors has been predicted by several band calculations carried out for $\mathrm{LaFeAsO},{ }^{3)} \mathrm{BaFe}_{2} \mathrm{As}_{2},{ }^{7)}$ and $\left.\mathrm{Fe}(\mathrm{Se}, \mathrm{Te}) .{ }^{8}\right)$ In $\mathrm{LaFeAsO}$ and $\mathrm{BaFe}_{2} \mathrm{As}_{2}$, superconductivity is induced by chemical substitution of elements with different

${ }^{*}$ E-mail address: ichiro@criepi.denken.or.jp 
valence number, and a rigid-band picture properly works. ${ }^{9-11)}$ Isovalent Co substitution of $\mathrm{Fe}$ in $\mathrm{BaFe}_{2} \mathrm{As}_{2}$ is also considered to shift the Fermi level, and thus works as an electron doping to $\mathrm{BaFe}_{2} \mathrm{As}_{2} .{ }^{12)}$ In contrast to them, the role of chemical substitution in iron chalcogenides has remained unclear. Se and Te take the same valence state, and the band structures are quite similar to each other. ${ }^{8)}$ Hall effect measurements are one of the powerful techniques to trace how the electronic state changes from FeTe with Se substitution. ${ }^{13)}$ In this letter, we report on the detailed Hall-effect measurements in the parent compound FeTe thin films, and try to apply two-band Drude picture to demonstrate an interplay of electrons and holes using resistivity and Hall coefficients data. Although the band calculation predicts the sufficient amount of electron and hole density in FeTe, difference of the magnitude of mobility is remarkable not only in the paramagnetic (PM) but also in the AFM states, which suggests the absence of interplay between two types of carriers.

Thin film samples were prepared by pulsed-laser deposition using a stoichiometric FeTe sintered target. ${ }^{13,14)}$ We choose $\mathrm{MgO}(100)$ as a substrate following the results of substrate selection in the growth of $\mathrm{Fe}\left(\mathrm{Se}_{0.5} \mathrm{Te}_{0.5}\right)$ thin films. ${ }^{15)}$ A metal mask was used to make the film in a six-terminal shape. Three FeTe films with different thicknesses are compared in the present study: The thickness of samples A, B, and C is 40, 400, and $165 \mathrm{~nm}$, respectively. The crystal structure is characterized by an x-ray diffraction and transmission electron microscopy (TEM), and the charge transport is characterized by Physical Properties Measurement System (Quantum Design).

Figure 1(a) shows x-ray diffractions of the three samples. Sample C shows almost perfect $c$-axis orientation, while the other two contain the (101)-oriented domains. In sample A, the 101 reflection is far intense than the $00 l$ reflections. In sample B the intensity of the $00 l$ reflections becomes strong but still the portion of the (101)-oriented domains are not small. We should note, however, that even in sample A high-resolution transmission microscopy (TEM) observation demonstrates a highly c-axis oriented structure [Fig. 1(b)]. We can see a sharp interface between $\mathrm{MgO}$ and $\mathrm{FeTe}$ similar to the case of $\mathrm{Fe}\left(\mathrm{Se}_{0.5} \mathrm{Te}_{0.5}\right)$ films on $\left.\mathrm{MgO},{ }^{15}\right)$ but simultaneously some deteriorated layer is observed at the surface probably due to oxidation during long-time expose in the air. The calculated $c$-axis lengths from the $00 l$ reflections are $6.258 \AA, 6.300 \AA$, and $6.285 \AA$ for samples A, B, and C, respectively.

Samples A and B exhibit higher resistivities than sample C does as shown in Fig. 1(c), probably because of the admixture of the (101)-oriented domains. The difference is consistent with that $\rho$ along the FeTe layer is slightly lower than that perpendicular to it. It should be noted that $\rho$ of sample $\mathrm{C}$ almost equals to that of bulk single crystals ever reported, ${ }^{16)}$ indi- 
cating that sample $\mathrm{C}$ exhibits an intrinsic in-plane electric transport. Trace of the tetragonalto-monoclinic structural phase transition is not observed in samples B and C. In bulk samples, resistivity exhibits a discontinuous jump at $T_{t m}=68 \mathrm{~K},{ }^{16,17)}$ but samples $\mathrm{B}$ and $\mathrm{C}$ exhibit only a rounded peak in $\rho$ around $T=80 \mathrm{~K}$ followed by a rather smooth decrease to low temperatures as reported. ${ }^{18,19)}$ However, except for the absence of sharp drop at $T_{t m}$, overall temperature dependence of $\rho$ is similar between the films and bulk crystals. Thus, crossover from tetragonal to monoclinic phases is likely to take place, and hence, we safely assume that an AFM long-range order is present in samples $\mathrm{B}$ and $\mathrm{C}$ at low temperatures. In contrast to them, $\rho$ shows a steep drop at $T=57 \mathrm{~K}$ in sample A, indicating the presence of a first-order transition.

Another important feature is that sample A shows a steep drop of resistivity below $11 \mathrm{~K}$. This can be attributed to the onset of superconductivity, because a typical suppression of the transition temperature $\left(T_{c}\right)$ with external magnetic field is observed as shown in the inset of Fig 1(d). The superconductivity was less obvious immediately after the growth of films, but showed up after two months, and then became unchanged. Thus, this superconductivity seems to be due to an aging effect discussed for $\mathrm{Fe}(\mathrm{Te}, \mathrm{Se})$ polycrystalline samples. ${ }^{20-22)}$ However, we cannot exclude a possible epitaxial strain effect discussed by Han et al., ${ }^{19)}$ because the $c$-axis length is far shorter in sample A than in samples B and C. Whatever the reason is, our interest is focused on how the appearance of superconductivity is related to the normal-state electric transport.

The Hall effect is investigated in the same way as was performed to $\mathrm{Fe}(\mathrm{Se}, \mathrm{Te})$ thin films. ${ }^{13)}$ We first measure the transverse resistivity by sweeping the magnetic field between $-9 \mathrm{~T} \leq$ $\mu_{0} H \leq 9 \mathrm{~T}$ applied normal to the film surface, and then extract an asymmetric component to determine the Hall resistivity $\rho_{x y}$. Figures 2(a) - 2(c) show the field dependence of $\rho_{x y}$ at different temperatures. We find that samples A and B show steep increase up to $\mu_{0} H=2 \mathrm{~T}$, which is due to an anomalous Hall effect (AHE). $\rho_{x y}$ can be expressed as $\rho_{x y}=B \cdot R_{H}+\rho_{s}$, where $B$ is the magnetic flux density, and $\rho_{s}$ is the anomalous term of the Hall resistivity. On the other hand, no trace of AHE is detected in sample C evoking that AHE is not a phenomenon intrinsic to FeTe. The inset of Fig. 2(d) shows $B$ dependence of $d \rho_{x y} / d B$. This gives a rough measure to determine which field range is appropriate for the linear fitting to $\rho_{x y}$, and we determine to fit the data at $2 \leq \mu_{0} H \leq 4 \mathrm{~T}$. It should be noted that the degree of linearity in high-field region is not significantly different among the three films, which indicates that the effect of AHE is limited in the weak-field region.

The field-dependence of $\rho_{x y}$ at low temperatures shows contrasting results from 
$\mathrm{Fe}\left(\mathrm{Se}_{0.5} \mathrm{Te}_{0.5}\right)$. In our previous study of $\mathrm{Fe}\left(\mathrm{Se}_{0.5} \mathrm{Te}_{0.5}\right)$ thin films, ${ }^{13)} \rho_{x y}$ exhibits a strong nonlinearity to the field at low temperatures, which was considered as a strong evidence of the collapse of the condition of compensated metals, i.e. electron density $\neq$ hole density. Such behavior is hardly observed in FeTe except for the data at limited temperature regions; one is around $T=40-70 \mathrm{~K}$ where the slope of $\rho_{x y}$ changes from positive to negative, and the other is $T=10 \mathrm{~K}$ for sample A only. There are two possible reasons for this strong suppression of nonlinearity. One is that electron density is equal to hole density, and the other is that the mobility of one-type carrier is far larger than that of the other carrier. We will discuss it later.

Temperature dependence of $R_{H}$ is summarized in Fig. 2(d). It is surprising that all three films follow almost the same lines in the high-temperature region, which indicates that the mixture of the (101)-oriented domain does not give a strong influence to $R_{H}$. The inset of Fig. 2(d) demonstrates that at $T=300 \mathrm{~K}$ the slope of $\rho_{x y}$ is similar to each other in spite of the difference of $\rho_{s}$. The deviation from the high-temperature trend becomes obvious below $100 \mathrm{~K}$. $R_{H}$ 's start to decrease rapidly, change sign from positive to negative, and then are roughly saturated below $30 \mathrm{~K}$. The value at the lowest temperature $T=10 \mathrm{~K}$ is $-6.72 \times 10^{-10} \mathrm{~m}^{3} / \mathrm{C}$, $-2.38 \times 10^{-9} \mathrm{~m}^{3} / \mathrm{C}$, and $-2.71 \times 10^{-9} \mathrm{~m}^{3} / \mathrm{C}$ for samples $\mathrm{A}, \mathrm{B}$, and $\mathrm{C}$, respectively. The values for samples $\mathrm{B}$ and $\mathrm{C}$ are roughly consistent with the recently reported numbers in bulk single crystals. $^{23)}$

In order to obtain further insight to the behavior of electrons and holes, it is useful to evaluate the mobility of carriers. However, it is unrealistic to directly deal with the five conduction bands of FeTe. We thus adopted a reduced two-band model containing one electron band and one hole band. In the two-band Drude model, resistivity and Hall coefficients can be described as $\rho=1 / e\left(\mu_{h} n_{h}+\mu_{e} n_{e}\right)$ and $R_{H}=\left(\mu_{h}^{2} n_{h}-\mu_{e}^{2} n_{e}\right) / e\left(\mu_{h} n_{h}+\mu_{e} n_{e}\right)^{2}$, where $\mu_{h}, \mu_{e}$, $n_{h}$, and $n_{e}$ are hole mobility, electron mobility, hole density, and electron density, respectively. Once $n_{h}$ and $n_{e}$ are given, we can calculate $\mu_{h}$ and $\mu_{e}$. As was first predicted theoretically, ${ }^{24)}$ and was later confirmed by neutron-diffraction studies, ${ }^{25,26)}$ FeTe has a bicollinear AFM order in low-temperature region. Therefore, we may apply the calculated carrier densities by Ma et al. ${ }^{24)}$ to our mobility estimation, in which $n_{h}\left(n_{e}\right)=2.26(2.38) \times 10^{27} \mathrm{~m}^{-3}$ are reported. Ma et al. also reported the carrier densities in the nonmagnetic state as $n_{h}$ $=n_{e}=4.77 \times 10^{27} \mathrm{~m}^{-3}$ (corresponding to a compensated metal), ${ }^{24}$ ) and at first glance these values correspond to the high-temperature state. However, the presence of free localized spins above $T_{t m}$ has been reported by magnetic susceptibility. ${ }^{27,28)}$ This means that the hightemperature region should be regarded as a Curie-Weiss type paramagnetic state, and hence those calculated for the nonmagnetic state are likely overestimated. We thus need to apply 
the carrier densities calculated for the paramagnetic state, and use the following values $n_{h}$ $\left(n_{e}\right)=1.07(1.26) \times 10^{27} \mathrm{~m}^{-3}$. which will appear elsewhere in near future. ${ }^{29)}$

Calculated mobility is summarized in Figs. 3(a)-(c). At high-temperature ( $T \geq 70 \mathrm{~K})$, we plot $\mu_{h}$ and $\mu_{e}$ not only for the paramagnetic case but also the nonmagnetic case for comparison. It is easily seen that the $\mu_{e}$ takes negative values in the whole temperature region in common to all the films, and thus this assumption looks unrealistic. In the paramagnetic case, we obtained rather reasonable values of mobilities, and we discuss them hereafter. The electric transport is dominated by holes at high temperatures; $\mu_{h}$ exceeds $\approx 4 \times 10^{-4} \mathrm{~m}^{2} \mathrm{~V}^{-1} \mathrm{~s}^{-1}$ for samples $\mathrm{A}$ and $\mathrm{B}$, while $\mu_{h}$ of sample $\mathrm{C}$ reaches almost $8 \times 10^{-4} \mathrm{~m}^{2} \mathrm{~V}^{-1} \mathrm{~s}^{-1}$, which is the main reason of the lower resistivity of sample C. $\mu_{e}$ 's commonly show small values for three samples; $\mu_{e}$ 's are approximately half of $\mu_{h}$, indicating that the contribution of $n$-type carrier is strongly suppressed in the paramagnetic (PM) state. This is surprising because the band calculation predict almost similar band mass for both the electron pocket around $M$ point and the hole pocket around $\Gamma$ point. ${ }^{8,24)}$ We may infer that this large difference in the character of $p$ - and $n$-type carriers is dependent on the more detailed structure of the Fermi surfaces, which will be clarified with ARPES and/or another methods.

The dominancy of holes is completely interchanged when the antiferromagnetic long-range order is evolved. Once FeTe goes into the AFM state, $\mu_{h}$ starts decreasing and simultaneously $\mu_{e}$ starts increasing. Strikingly, $\mu_{h}$ is completely suppressed to zero in samples B and C, which indicates that the $p$ type carrier has been almost localized. On the other hand, the evolution of $\mu_{e}$ is remarkable, and its magnitude exceeds the value of $\mu_{h}$ in the PM state. This large enhancement of $\mu_{e}$ is the main reason of metallic, $(d \rho / d T>0)$, conduction in the AFM state. Another important finding is that the suppression of $\mu_{h}$ is less emphasized in sample A. The suppression of $\mu_{h}$ actually occurs in sample A, but the minimum value of $\mu_{h}$ remains at sufficiently high value as $1.8 \times 10^{-4} \mathrm{~m}^{2} \mathrm{~V}^{-1} \mathrm{~s}^{-1}$, which is comparable to $\mu_{e}$. Therefore, we may expect in sample A that both electrons and holes coexist as itinerant carriers even in the AFM state.

The mobility analysis indicates that $\mu_{h}$ remains finite in superconducting sample while it goes to almost zero in nonsuperconducting samples. In other words, sufficient itinerancy is necessary for the occurrence of superconductivity in both electron and hole bands. This result prefers a scenario of pairing mechanism requiring an interband scattering between $p$ - and $n$-type Fermi surfaces, and hence supports either $s_{++}$-wave or $s_{ \pm}$-wave pairing states, ${ }^{4,5,30}$ ) while it may exclude the possibility of simple anisotropic $s$ - and $d$-wave pairing state in which gap opens independently on each Fermi surface. One may claim that the strong suppression 
of $\mu_{h}$ to zero is just an artifact of the calculations. However, we have confirmed that a slight change in the carrier density never alters the tendency that superconducting sample exhibits higher hole mobility than that nonsuperconducting one does, and hence, the correlation of superconductivity with the itinerancy of holes and electrons seems to be robust. It should be noted that our analysis taking only two bands into account does not loose generality for the real five band transport. We should emphasize again that the present results discuss an extreme case where $\mu_{h}$ is almost zero at low temperatures. In this case, we may safely conclude that all holes are not mobile, and the qualitative discussion carried out before is still valid.

In conclusion, we measured the Hall effect for nonsuperconducting and superconducting FeTe thin films in detail. Temperature dependence of the Hall coefficients is analyzed by a semiclassical two-band Drude model with the aid of calculated carrier density. The estimated mobility of $n$ - and $p$-type carriers demonstrates a remarkable interchange across the antiferromagnetic transition. In the antiferromagnetic state, the mobility of $p$-type carriers of nonsuperconducting FeTe is suppressed to almost zero, while it remains finite in superconducting FeTe, which indicates that the necessity condition for the occurrence of superconductivity is that both electrons and holes remains itinerant.

The authors are grateful to Z. -Y. Lu and Tao Xiang for sharing with us the unpublished data prior to publication. We also thank H. Kontani, T. Tohyama, and K. Ohgushi fruitful discussions. 


\section{References}

1) Y. Kamihara, T. Watanabe, M. Hirano, and H. Hosono: J. Am. Chem. Soc. 130 (2008) 3296.

2) F. C. Hsu, J. Y. Luo, K. W. Yeh, T. K. Chen, T. W. Huang, P. M. Wu, Y. C. Lee, Y. L. Huang, Y. Y. Chu, D. C. Yan, and M. K. Wu: Proc. Natl. Acad. Sci. USA 105 (2008) 14262.

3) D.J. Singh and M.-H. Du: Phys. Rev. Lett. 100 (2008) 237003.

4) I.I. Mazin, D.J. Singh, M.D. Johannes, and M.H. Du: Phys. Rev. Lett. 101 (2008) 057003.

5) K. Kuroki, S. Onari, R. Arita, H. Usui, Y. Tanaka, H. Kontani, and H. Aoki: Phys. Rev. Lett. 101 (2008) 087004.

6) M. Rotter, M. Tegel, D. Johrendt, I. Schellenberg, W. Hermes, and R. Pöttgen: Phys. Rev. B 78 (2008) 020503(R).

7) D.J. Singh: Phys. Rev. B 78 (2008) 094511.

8) A. Subedi, L. Zhang, D. J. Singh, and M. H. Du: Phys. Rev. B 78 (2008) 134514.

9) Y. Kohama, Y. Kamihara, S. A. Baily, L. Civale, S. C. Riggs, F. F. Balakirev, T. Atake, M. Jaime, M. Hirano, and H. Hosono: Phys. Rev. B 79 (2009) 144527.

10) F. Rullier-Albenque, D. Colson, A. Forget, and H. Alloul: Phys. Rev. Lett. 103 (2009) 057001.

11) L. Fang, H. Luo, P. Cheng, Z. Wang, Y. Jia, G. Mu, B. Shen, I.I. Mazin, L. Shan, C. Ren, and H. H. Wen: Phys. Rev. B 80 (2009) 140508(R).

12) N. Katayama, Y. Kiuchi, Y. Matsushita, and K. Ohgushi: J. Phys. Soc. Jpn. 78 (2009) 123702.

13) I. Tsukada, M. Hanawa, S. Komiya, T. Akiike, R. Tanaka, Y. Imai, and A. Maeda: Phys. Rev. B 81 (2010) 054515.

14) Y. Imai, R. Tanaka, T. Akiike, M. Hanawa, I. Tsukada, and A. Maeda: Jpn. J. Appl. Phys. 49 (2010) 023101.

15) Y. Imai, R. Tanaka, T. Akiike, M. Hanawa, I. Tsukada, A. Ichinose, A. Maeda, K. Hikage, T. Kawaguchi, and H. Ikuta: Appl. Phys. Express 3 (2010) 043102.

16) T. Noji, T. Suzuki, H. Abe, T. Adachi, M. Kato, and Y. Koike: J. Phys. Soc. Jpn. 79 (2010) 084711.

17) Y. Mizuguchi, F. Tomioka, S. Tsuda, T. Yamaguchi, and Y. Takano: Appl. Phys. Lett. 94 (2009) 012503.

18) P. Mele, K. Matsumoto, Y. Haruyama, M. Mukaida, Y. Yoshida, Y. Ichino, T. Kiss, and A. Ichinose: Superconduct. Sci. Technol. 23 (2010) 052001.

19) Y. Han, W.Y. Li, L.X. Cao, X.Y. Wang, B. Xu, B.R. Zhao, Y.Q. Guo, and J.L. Yang: Phys. Rev. Lett. 104 (2010) 017003.

20) Y. Mizuguchi, K. Deguchi, S. Tsuda, T. Yamaguchi, and Y. Takano: Phys. Rev. B 81 (2010) 214510.

21) Y. Mizuguchi, K. Deguchi, S. Tsuda, T. Yamaguchi, and Y. Takano: Europhys. Lett. 90 (2010) 57002.

$22)$ Y. Nie, D. Telesca, J. I. Budnick, B. Sinkovic, and B. O. Wells: Phys. Rev. B 82 (2010) 020508(R).

23) T. J. Liu, J. Hu, B. Qian, D. Fobes, Z. Q. Mao, W. Bao, M. Reehuis, S. A. J. Kimber, K. Prokes, S. Matas, D. N. Argyriou, A. Hiess, A. Rotaru, H. Pham, L. Spinu, Y. Qiu, V. Thampy, A. T. Savici, J. A. Rodrigues, and C. Broholm: Nature Mater. 9 (2010) 716.

24) F. Ma, W. Ji, J. Hu, Z.-Y. Lu, and T. Xiang: Phys. Rev. Lett. 102 (2009) 177003.

25) W. Bao, Y. Qiu, Q. Huang, M. A. Green, P. Zajdel, M. R. Fitzsimmons, M. Zhemenkov, S. Chang, M. Fang, B. Qian, E. K. Vehstedt, J. Yang, H. M. Pham, L. Spinu, and Z. Q. Mao: Phys. Rev. Lett. 102 (2009) 247001. 
26) S. Li, C. delaCruz, Q. Huang, Y. Chen, J.W. Lynn, J. Hu, Y.-L. Huang, F.-C. Hsu, K.-W. Yeh, M. K. Wu, and P. Dai: Phys. Rev. B 79 (2009) 054503.

27) Y. Xia, D. Qian, L. Wray, D. Hsieh, G. F. Chen, J. L. Luo, N. L. Wang, and M. Z. Hasan: Phy. Rev. Lett. 103 (2009) 037002.

28) C. Michioka, H. Ohta, M. Matsui, J. Yang, K. Yoshimura, and M. Fang: Phys. Rev. B 82 (2010) 064506 .

29) Z. Y. Lu: private communication.

30) H. Kontani and S. Onari: Phys. Rev. Lett. 104 (2010) 157001. 

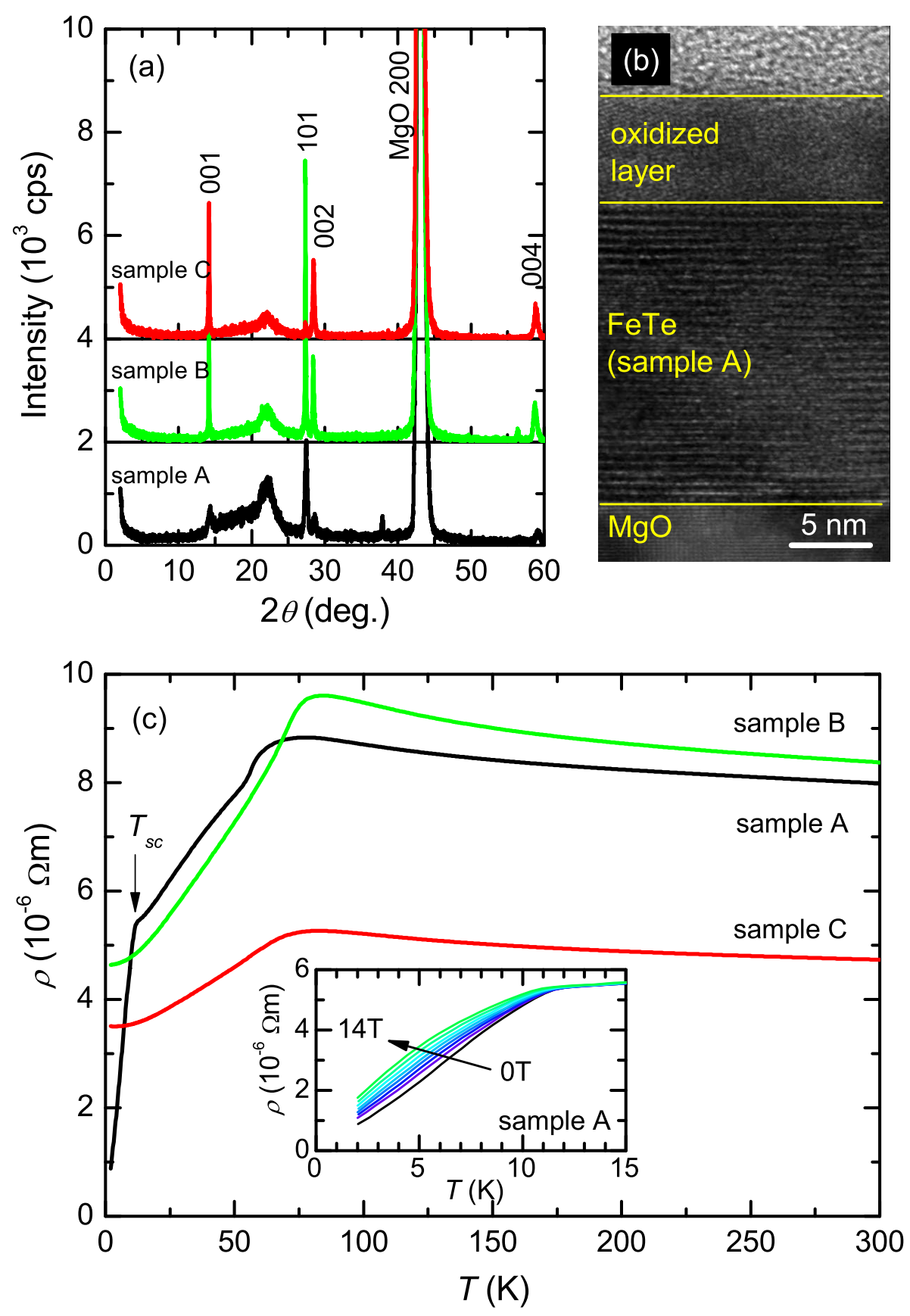

Fig. 1. (Color online) (a) X-ray diffraction of the three films. (b) Cross section TEM image of sample A. (c) Temperature dependence of resistivity of the three films. The inset shows magnetic field dependence of resistivity of sample A. 

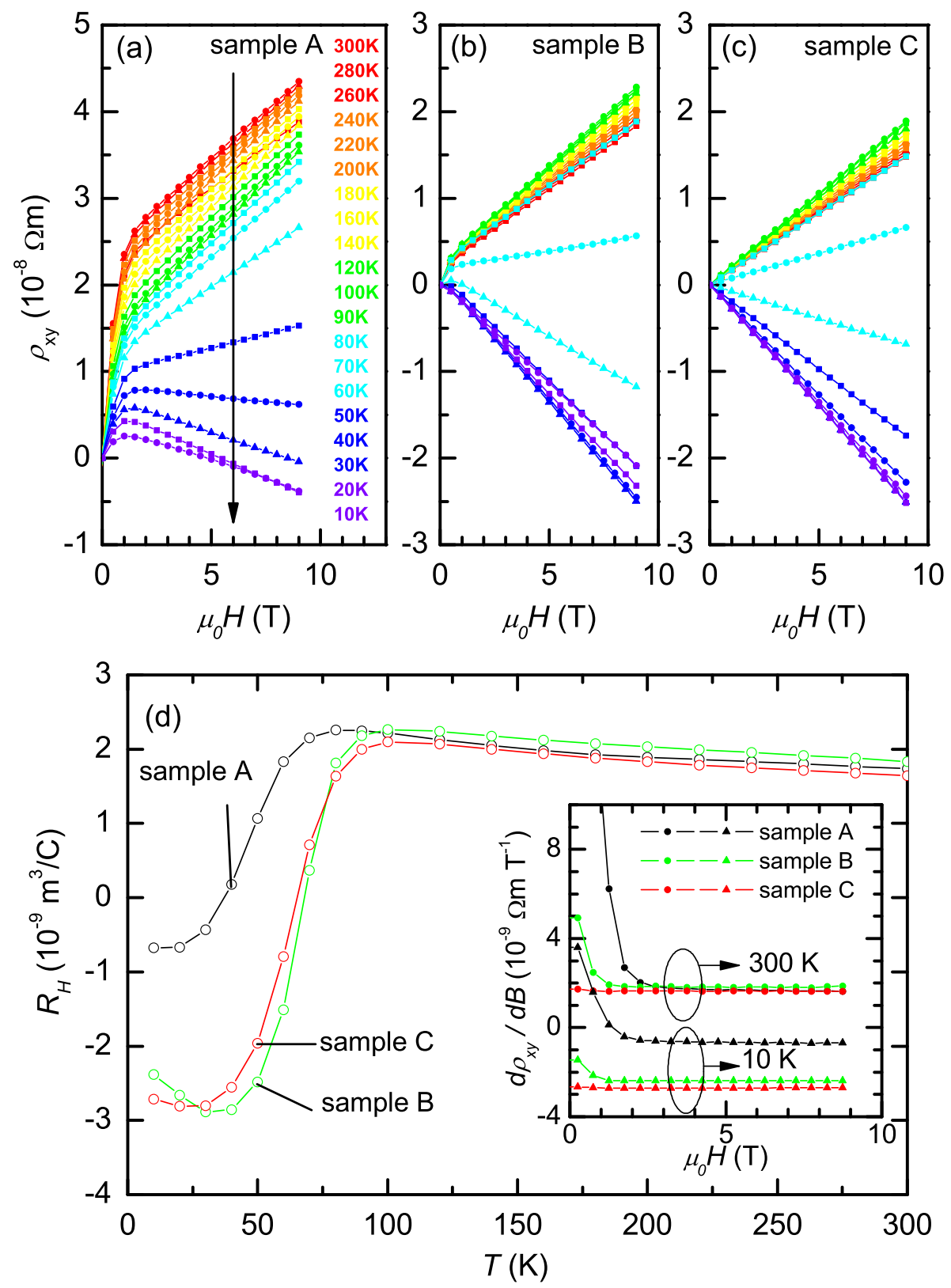

Fig. 2. (Color online) (a)-(c) Field dependence of $\rho_{x y}$ at different temperatures for the three films. Finite contribution of the anomalous Hall effect is detected in $\rho_{x y}$ of samples A and B, while $\rho_{x y}$ is almost linear to $B$ in sample C. (d) Temperature dependence of $R_{H}$ for the three films. The inset shows $d \rho_{x y} / d B$ at $T=300$ and $10 \mathrm{~K}$. 

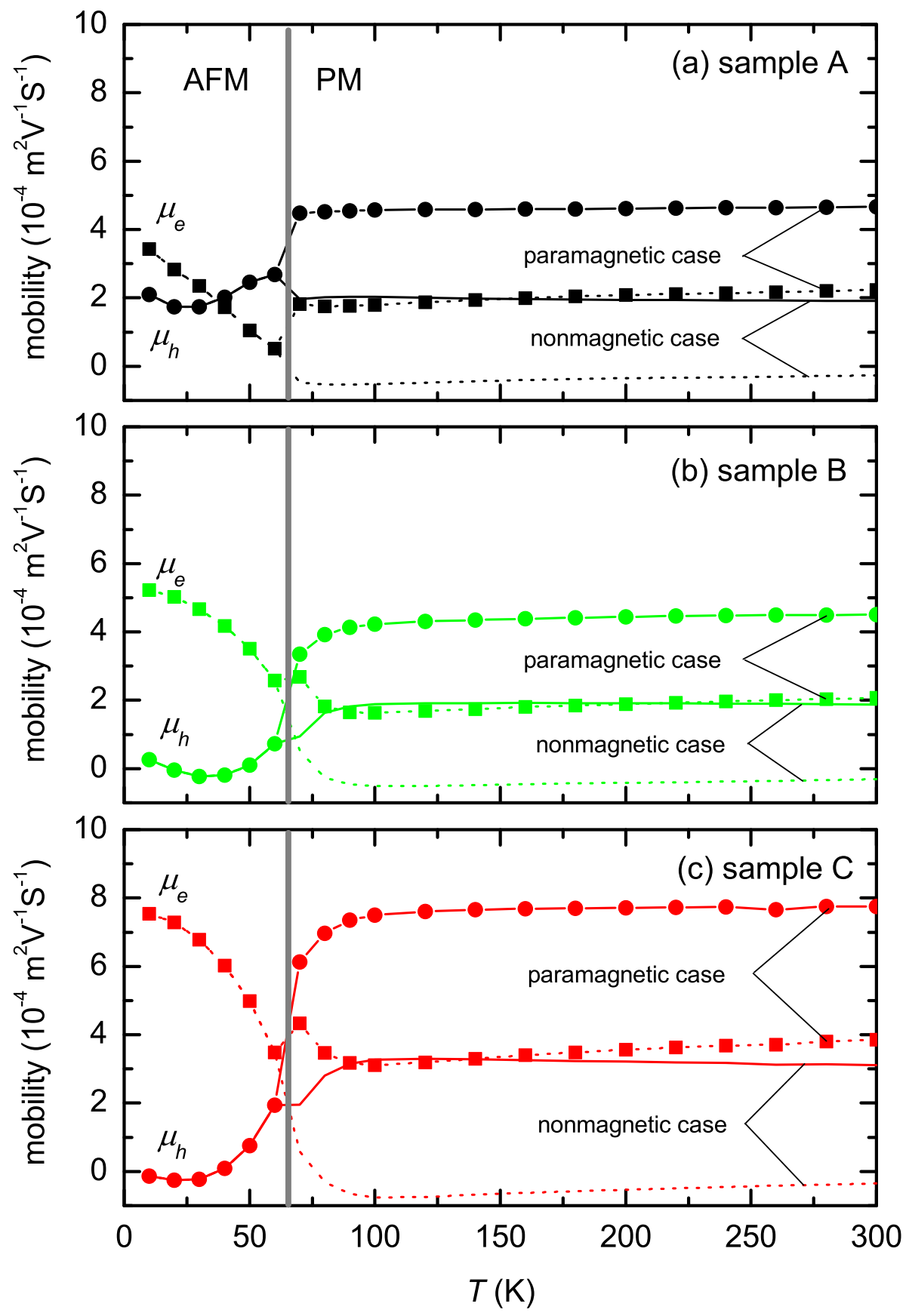

Fig. 3. (Color online) (a)-(c) Mobility of holes and electrons in three cases; $n_{h}\left(n_{e}\right)=2.26(2.38) \times$ $10^{27} \mathrm{~m}^{-3}$ in the bicollinear AFM phase at $T \leq 60 \mathrm{~K}$, while at $T \geq 70 \mathrm{~K} n_{h}\left(n_{e}\right)=1.07(1.26)$ $\times 10^{27} \mathrm{~m}^{-3}$ for the paramagnetic case, and $n_{h}=n_{e}=4.77 \times 10^{27} \mathrm{~m}^{-3}$ in nonmagnetic case are assumed. 